April 1, 2013

\title{
The Asymmetric Simple Exclusion Process with an Open Boundary
}

\author{
Craig A. Tracy \\ Department of Mathematics \\ University of California \\ Davis, CA 95616, USA \\ Harold Widom \\ Department of Mathematics \\ University of California \\ Santa Cruz, CA 95064, USA
}

\section{Introduction}

In previous work [7] the authors considered the asymmetric simple exclusion process (ASEP) where particles are confined to the nonnegative integers $\mathbb{Z}^{+}=\{0,1,2, \ldots\}$. Each particle waits exponential time, and then with probability $p$ it moves one step to the right if the site is unoccupied, otherwise it does not move; and with probability $q=1-p$ a particle not at 0 moves one step to the left if the site is unoccupied, otherwise it does not move. For $n$-particle ASEP a possible cofigurations is

$$
\mathbf{x}=\left\{x_{1}, \ldots, x_{n}\right\}, \quad\left(0 \leq x_{1}<\cdots<x_{n}\right) .
$$

The $x_{i}$ are the occupied sites. We denote by $\mathcal{X}_{n}$ the set of possible configurations for $n$-particle ASEP, and by $\mathfrak{p}_{n}(\mathbf{x}, \mathbf{y} ; t)$ the probability that at time $t$ the system is in configuration $\mathbf{x}$ given that initially it was in configuration $\mathbf{y}$. (We shall drop the subscript " $n$ " when it is understood.)

In [7] a formula was found for this probability. It was the sum over the Weyl group $\mathbb{B}_{n}$ of multiple integrals. (For ASEP on $\mathbb{Z}$ it was a sum over the permutation group $\mathbb{S}_{n}[5,6]$.)

Here we consider the ASEP on $\mathbb{Z}^{+}$with an open boundary at zero. The stationary measure for ASEP on the finite lattice $[1, L]$ or on the semi-infinite lattice $\mathbb{Z}^{+}$with boundaries connected to reservoirs has been the subject of much research starting with Derrida et al. [1]. We refer the reader to the recent work of Sasamoto and Williams [4] for an up-to-date account of these developments. Here we consider the time-dependent properties of ASEP on $\mathbb{Z}^{+}$with an open boundary. Specifically, the point 0 is connected to a reservoir where a particle is injected into site 0 from the 
reservoir at a rate $\alpha$, assuming that the site 0 is empty, and a particle at site 0 is ejected into the reservoir at a rate $\beta$. Now the number of particles is not conserved and for ASEP with open boundary the configuration $\mathbf{x}$ may lie in $\mathcal{X}_{n}$ while $\mathbf{y}$ may lie in $\mathcal{X}_{m}$ with $m \neq n$.

We find an infinite tri-diagonal matrix with operator entries in which the Laplace transforms of the probabilities can be read off from the entries of the inverse matrix. When either $\alpha=0$ or $\beta=0$ the matrix is triangular and so the inverse can be computed more explicitly. The result is obtained by solving a system of differential equations for the probabilities. The final formulas involve inverses of operators with kernels the Laplace transforms of certain $\mathfrak{p}(\mathbf{x}, \mathbf{y} ; t)$ obtained in [7].

There are two special cases in which the results are more explicit. For TASEP with $p=1$, the inverse operator is computable in terms of $\mathfrak{p}(\mathbf{x}, \mathbf{y} ; t)$ itself, and the probabilities are given in terms of certain determinants. For $\operatorname{SSEP}(p=q)$ and general $\alpha$ and $\beta$ we find formulas analogous to the ones described above for the probability that sites $x_{1}, \ldots, x_{n}$ are occupied. This is for infinite systems as well as finite ones.

We state the formulas for $\mathfrak{p}(\mathbf{x}, \mathbf{y} ; t)$ in the Appendix.

\section{Statement of results}

We denote by $\mathcal{E}_{n}$ the Lebesgue space $L^{1}\left(\mathcal{X}_{n}\right)$. From the fact that

$$
\sum_{\mathbf{x} \in \mathcal{X}_{n}} \mathfrak{p}(\mathbf{x}, \mathbf{y} ; t)=1
$$

for each $\mathbf{y}$, it follows that the operator on $\mathcal{E}_{n}$ with this kernel is bounded with norm one. We denote the Laplace transform of $\mathfrak{p}(\mathbf{x}, \mathbf{y} ; t)$ by $\widehat{\mathfrak{p}}(\mathbf{x}, \mathbf{y} ; s)$ :

$$
\widehat{\mathfrak{p}}(\mathbf{x}, \mathbf{y} ; s)=\int_{0}^{\infty} \mathfrak{p}(\mathbf{x}, \mathbf{y} ; t) e^{-s t} d t .
$$

The operator with this kernel is bounded on $\mathcal{E}_{n}$ with norm at most $(\operatorname{Re} s)^{-1}$. We denote it by $L_{n}(s) 1$ In the results stated below it is tacitly assumed that $\operatorname{Re} s$ is sufficiently large.

Now for ASEP with open boundary at zero, we define $P_{n}(\mathbf{x} ; t)$ to be the probability that the system is in configuration $\mathrm{x} \in \mathcal{X}_{n}$ at time $t$. (We shall usually drop the " $\mathrm{x}$ " in the notation, and do not specify an initial configuration.) We denote its Laplace transform by $\widehat{P}_{n}(s)$.

We define vector functions

$$
\widehat{P}(s)=\left(\widehat{P}_{n}(s)\right)_{n \geq 0}, \quad P(0)=\left(P_{n}(0)\right)_{n \geq 0},
$$

\footnotetext{
${ }^{1}$ When $n=0$ we interpret $\mathfrak{p}(\mathbf{x}, \mathbf{y} ; t)$ as 1 , and so $L_{0}(s)$ is multiplication by $s^{-1}$.
} 
belonging to the direct $\operatorname{sum} \sum_{n=0}^{\infty} \mathcal{E}_{n}$.

We define operators $A_{n}: \mathcal{E}_{n-1} \rightarrow \mathcal{E}_{n}$ and $B_{n}: \mathcal{E}_{n+1} \rightarrow \mathcal{E}_{n}$ by

$$
\begin{gathered}
\left(A_{n} F\right)\left(x_{1}, \ldots, x_{n}\right)=\delta\left(x_{1}\right) F\left(x_{2}, \ldots, x_{n}\right), \\
\left(B_{n} F\right)\left(x_{1}, \ldots, x_{n}\right)=\left(1-\delta\left(x_{1}\right)\right) F\left(0, x_{1}, \ldots, x_{n}\right), 2
\end{gathered}
$$

Then we define matrices $\delta, L(s), A, B$, with operator entries, acting on $\sum_{n=0}^{\infty} \mathcal{E}_{n}$. The first is diagonal with $(n, n)$-entry multiplication by $\delta\left(x_{1}\right)$, the second is diagonal with $n, n$-entry $L_{n}(s)$, the third is subdiagonal (one diagonal below the main diagonal) with $n, n-1$-entry $A_{n}$, and the last is superdiagonal (one diagonal above the main diagonal) with $n, n+1$-entry $B_{n}$.

Theorem 1. With this notation we have

$$
\widehat{P}(s-\alpha)=(I-L(s)((\alpha-\beta) \delta+\alpha A+\beta B))^{-1} L(s) P(0) .
$$

There are expressions for the entries of the inverse operator as infinite series of products. But when either $\beta=0$ or $\alpha=0$ the operator has only one subdiagonal or one superdiagonal and each entry of the inverse is a single product. We state the results as recursion formulas. Define

$$
M_{n}(s)=\left(I-(\alpha-\beta) L_{n}(s) \delta\right)^{-1}
$$

Corollary 1.1. Suppose $\beta=0$ and that initially there are $k$ particles at $\mathbf{y} \in \mathcal{X}_{k}$. Then

$$
\widehat{P}_{k}(s-\alpha)=M_{k}(s) L_{k}(s) \delta_{\mathbf{y}}, 3
$$

and when $n>k$

$$
\widehat{P}_{n}(s-\alpha)=\alpha M_{n}(s) L_{n}(s) A_{n} \widehat{P}_{n-1}(s-\alpha)
$$

Corollary 1.2. Suppose $\alpha=0$ and that initially there are $k$ particles at $\mathbf{y} \in \mathcal{X}_{k}$. Then

$$
\widehat{P}_{k}(s)=M_{k}(s) L_{k}(s) \delta_{\mathbf{y}}
$$

and when $n<k$

$$
\widehat{P}_{n}(s)=\beta M_{n}(s) L_{n}(s) B_{n} \widehat{P}_{n+1}(s) .
$$

In connection with the corollaries we show the following.

\footnotetext{
${ }^{2}$ When $n=0$ we interpret $\delta\left(x_{1}\right)$ as zero. In particular $A_{0}=0$ and $B_{0} F=F(0)$.

${ }^{3}$ This is interpreted as $s^{-1}$ when $k=0$.
} 
Remark 1.1. The operators appearing in the inverses can be replaced by lowerdimensional ones. This will be useful for computation. Define

$$
\mathcal{X}_{n}^{+}=\left\{\left\{x_{1}, \ldots, x_{n}\right\} \in \mathcal{X}_{n}: x_{1}>0\right\}, \quad \mathcal{E}_{n}^{+}=L^{1}\left(\mathcal{X}_{n}^{+}\right),
$$

and then operators:

$L_{n-1}^{0}(s): \mathcal{E}_{n-1}^{+} \rightarrow \mathcal{E}_{n-1}^{+}$with kernel $\widehat{\mathfrak{p}}((0, \mathbf{x}),(0, \mathbf{y}) ; s)$,

$L_{n, n-1}^{0}(s): \mathcal{E}_{n-1}^{+} \rightarrow \mathcal{E}_{n}$ with kernel $\widehat{\mathfrak{p}}(\mathbf{x},(0, \mathbf{y}) ; s)$,

$L_{n-1, n}^{0}(s): \mathcal{E}_{n} \rightarrow \mathcal{E}_{n-1}^{+}$with kernel $\widehat{\mathfrak{p}}((0, \mathbf{x}), \mathbf{y} ; s)$.

(a) The operator $M_{n}(s) L_{n}(s) A_{n}: \mathcal{E}_{n-1} \rightarrow \mathcal{E}_{n}$ in Corollary 1.1 is equal to

$$
L_{n, n-1}^{0}(s)\left(I-(\alpha-\beta) L_{n-1}^{0}(s)\right)^{-1} R_{n-1}, 4
$$

where $R_{n-1}: \mathcal{E}_{n-1} \rightarrow \mathcal{E}_{n-1}^{+}$is the restriction operator.

(b) The operator $M_{k}(s) L_{k}(s): \mathcal{E}_{k} \rightarrow \mathcal{E}_{k}$ in Corollaries 1.1 and 1.2 is equal to

$$
L_{k}(s)+(\alpha-\beta) L_{k, k-1}^{0}(s)\left(I-(\alpha-\beta) L_{k-1}^{0}(s)\right)^{-1} L_{k-1, k}^{0}(s) .
$$

Remark 1.2. In the special case of TASEP when $p=1$ we have the simplification $\left(I-\alpha L_{n}^{0}(s)\right)^{-1}=I+\alpha L_{n}^{0}(s-\alpha)$.

In the case of SSEP $(p=q)$, even for infinitly many particles, there are formulas for correlations that are no more complicated when both $\alpha$ and $\beta$ are nonzero. For $\mathbf{x}=\left\{x_{1}, \ldots, x_{n}\right\} \in \mathcal{X}_{n}$ we define $\Psi_{n}(\mathbf{x} ; t)$ to be the probability that sites $x_{1}, \ldots, x_{n}$ are occupied at time $t$. We denote its Laplace transform by $\widehat{\Psi}_{n}(s)=\widehat{\Psi}_{n}(\mathbf{x} ; s)$ and introduce the vector functions

$$
\widehat{\Psi}(s)=\left(\widehat{\Psi}_{n}(s)\right)_{n \geq 0}, \quad \Psi(0)=\left(\Psi_{n}(0)\right)_{n \geq 0} \text { 占 }
$$

Let the operators $L_{n}(s)$ and $A_{n}$, and the matrices $\delta, L(s), A$ with operator entries be the same as before 6

Theorem 2. We have,

$$
\widehat{\Psi}(s)=(I+L(s)((\alpha+\beta) \delta-\alpha A))^{-1} L(s) \Psi(0) .
$$

\footnotetext{
${ }^{4}$ The $I$ here is the identity operator on $\mathcal{E}_{n-1}^{+}$while the $I$ in (5) is the identity operator on $\mathcal{E}_{n}$.

${ }^{5}$ We define $\Psi_{0}(t)=1$, and so $\widehat{\Psi}_{0}(s)=s^{-1}$.

${ }^{6}$ Now we define $\mathcal{E}_{n}=L^{\infty}\left(\mathcal{X}_{n}\right)$, and observe that by (10) and the symmetry of the kernel we have $\sum_{\mathbf{y} \in \mathcal{X}_{n}} \mathfrak{p}(\mathbf{x}, \mathbf{y} ; t)=1$ for each $\mathbf{x} \in \mathcal{X}_{n}$. It follows that $L_{n}(s)$ is a bounded operator on this $\mathcal{E}_{n}$ with norm at most $(\operatorname{Re} s)^{-1}$.
} 
We now set

$$
M_{n}(s)=\left(I+(\alpha+\beta) L_{n}(s) \delta\right)^{-1}
$$

Corollary 2.1. For $n>0$,

$$
\widehat{\Psi}_{n}(s)=\alpha M_{n}(s) L_{n}(s) A_{n} \widehat{\Psi}_{n-1}(s)+M_{n}(s) L_{n}(s) \Psi_{n}(0) .
$$

Corollary 2.2. In the case of Bernoulli initial condition with density $\rho$,

$$
\widehat{\Psi}_{n}(s)=\alpha M_{n}(s) L_{n}(s) A_{n} \widehat{\Psi}_{n-1}(s)+s^{-1} \rho^{n} M_{n}(s) 1,
$$

where " 1 " is the constant function on $\mathcal{X}_{n}$.

Corollary 2.3. When initially no sites are occupied,

$$
\widehat{\Psi}_{n}(s)=\alpha M_{n}(s) L_{n}(s) A_{n} \widehat{\Psi}_{n-1}(s) .
$$

The analogue of Remark 1.1 holds here.

From the abstract formulas, Theorems 1 and 2 and their corollaries, we derive some concrete results.

Suppose, in ASEP, that at time zero there is a single particle at $y$. From Corollaries 1.1 and 1.2 we show:

$\boldsymbol{\alpha}=\mathbf{0}$ : When $p>q$, with probability

$$
1-\frac{\beta\left(q^{-1}-1\right)^{-y}}{p-q+\beta}
$$

the particle is never ejected. When $p \leq q$, with probability one the particle will eventually be ejected. The expected value of the time this occurs is infinite when $p=q$, and when $p<q$ it is

$$
\frac{y+q / \beta}{q-p} .
$$

$\boldsymbol{\beta}=0$ : With probability one a second particle will eventually be injected. The expected value of the time at which this occurs is

$$
\frac{1}{\alpha}+\frac{\xi_{+}(\alpha)^{-y}}{q\left(\xi_{+}(\alpha)-1\right)-\alpha}
$$

where

$$
\xi_{+}(\alpha)=\frac{1}{2 q}\left(\alpha+1+\sqrt{(\alpha+1)^{2}-4 p q}\right)
$$


Combining Remark 1.2 with the determinant formula (24) for $\mathfrak{p}(\mathbf{x}, \mathbf{y} ; t)$ in TASEP it is practical to compute some exact results. Denote by $\mathcal{P}_{n}(t)$ the probability that starting with no particles at time 0 there are exactly $n$ particles at time $t$. Then

$$
\begin{gathered}
\mathcal{P}_{0}(t)=e^{-\alpha t} \\
\mathcal{P}_{1}(t)=\frac{\alpha}{1-\alpha}\left(t-\frac{\alpha}{1-\alpha}\right) e^{-\alpha t}+\frac{\alpha^{2}}{(1-\alpha)^{2}} e^{-t} \\
\mathcal{P}_{2}(t)=\left(\frac{\alpha^{2}}{2(1-\alpha)^{2}} t^{2}-\frac{\alpha^{2}}{(1-\alpha)^{3}} t+\frac{\alpha^{2}}{(1-\alpha)^{2}}\right) e^{-\alpha t} \\
+\left(\frac{\alpha^{2}}{2(1-\alpha)^{2}} t^{2}-\frac{\alpha\left(1-3 \alpha+\alpha^{2}\right)}{(1-\alpha)^{3}}+\frac{1-2 \alpha}{(1-\alpha)^{2}}\right) e^{-t}-e^{-(1+\alpha) t} .
\end{gathered}
$$

These are for $\alpha \neq 1$. When $\alpha=1$ there is no singularity; we take a limit and the formulas simplify.

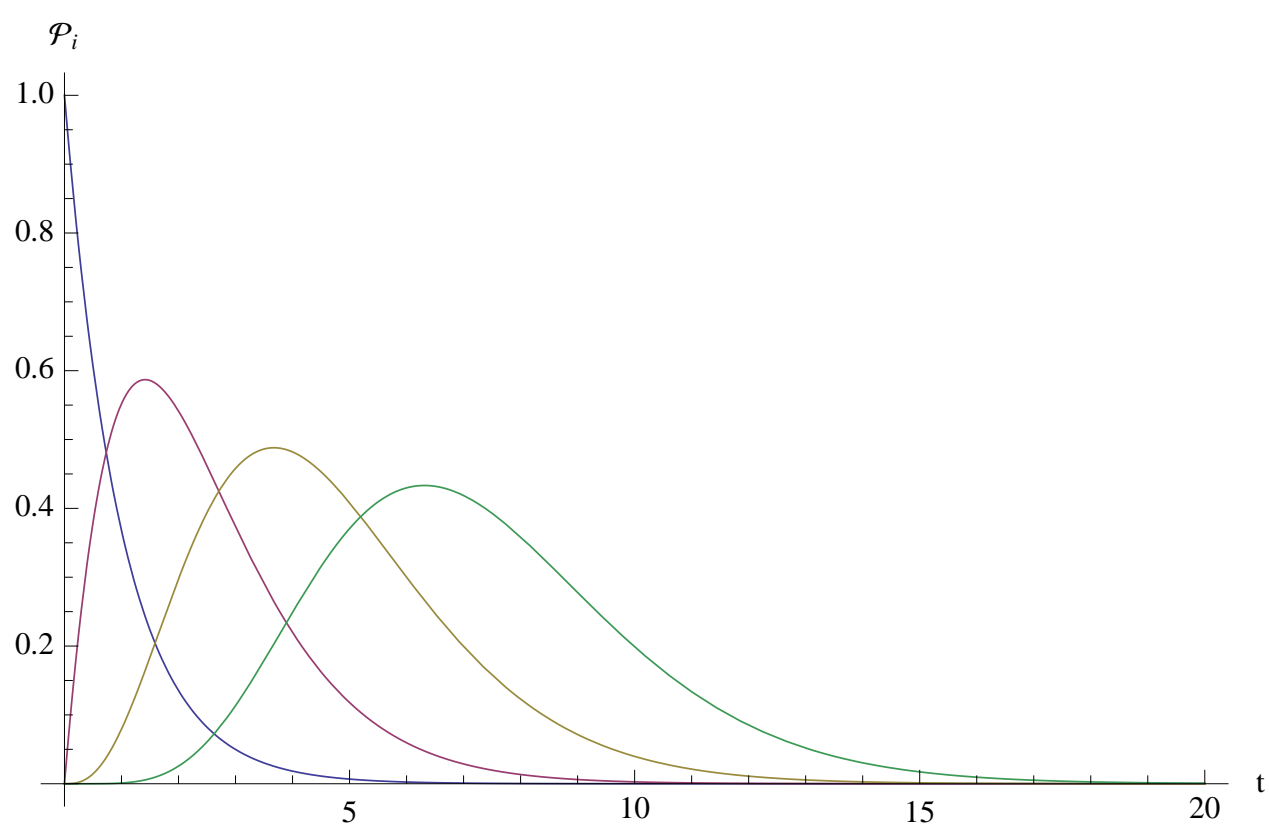

Figure 1: Plotted are the TASEP probabilities $\mathcal{P}_{n}(t)$ for $n=0,1,2,3$ with $\alpha=1$. Increasing $n$ moves the maximum to the right.

For SSEP we obtain the following consequence of Corollary 2.2. Define

$$
\Delta N(t)=\sum_{x \geq 0}\left(\eta_{x}(t)-\eta_{x}(0)\right)
$$


the net number of particles that have entered the system at time $t$. (Which may be negative.) With Bernoulli initial condition when $\left\langle\eta_{x}(0)\right\rangle=\rho$, we show for the expected value that

$$
\langle\Delta N(t)\rangle \sim \sqrt{\frac{2}{\pi}} \frac{\alpha-(\alpha+\beta) \rho}{\alpha+\beta} t^{1 / 2} \text { as } t \rightarrow \infty .7
$$

By a laborious computation (not included) we can show that the second moment of $\Delta N(t)$ is finite.

In the special case where initially there are no occupied sites, we use Corollary 2.3 and present a not completely rigorous (to say the least) argument that

$$
\left\langle\Delta N(t)^{2}\right\rangle \sim \frac{2}{\pi} \frac{\alpha^{2}}{(\alpha+\beta)^{2}} t \quad \text { as } t \rightarrow \infty .
$$

Combining this with the first moment asymptotics when $\rho=0$ we conclude that the variance of $\Delta N(t) / t^{1 / 2}$ tends to zero as $t \rightarrow \infty \sqrt[8]{ }$ The derivation is quite long, but the result with the precise constants came out so nicely in the end that we could not resist including it.

\section{Proofs of the results}

\section{A. ASEP}

Proof of Theorem 1: The probability $\mathfrak{p}(\mathbf{x}, \mathbf{y} ; t)$ for $n$-particle ASEP on $\mathbb{Z}^{+}$is the solution of the differential equation

$$
\begin{aligned}
& \frac{d}{d t} u(X ; t)=\sum_{i=1}^{N}\left[p u\left(x_{i}-1\right)\left(1-\delta\left(x_{i}-x_{i-1}-1\right)\right)+q u\left(x_{i}+1\right)\left(1-\delta\left(x_{i+1}-x_{i}-1\right)\right)\right. \\
& \left.-p u\left(x_{i}\right)\left(1-\delta\left(x_{i+1}-x_{i}-1\right)\right)-q u\left(x_{i}\right)\left(1-\delta\left(x_{i}-x_{i-1}-1\right)\right)\right]+\left[q u\left(x_{1}\right)-p u\left(x_{1}-1\right)\right] \delta\left(x_{1}\right)
\end{aligned}
$$

that satisfies the initial condition

$$
u(\mathbf{x} ; 0)=\delta_{\mathbf{y}}(\mathbf{x})
$$

(In the $i$ th summand entry $i$ is displayed and entry $j$ is $x_{j}$ when $j \neq i$.)

If we denote by $\mathcal{Q}_{n}$ the operator given by the right side of the equation, then $\mathfrak{p}(\mathbf{x}, \mathbf{y} ; t)$ is the kernel of $e^{t \mathcal{Q}_{n}}$. Thus the equation is

$$
\frac{d \mathfrak{p}_{n}}{d t}=\mathcal{Q}_{n} \mathfrak{p}_{n}(\mathbf{x} ; t)
$$

\footnotetext{
${ }^{7}$ This result was obtained in [2] in the case $\beta=0$ and $\rho=0$.

${ }^{8}$ As predicted in [2] in the case $\beta=0$.
} 
where we have written $\mathfrak{p}_{n}(\mathbf{x} ; t)$ for $\mathfrak{p}(\mathbf{x}, \mathbf{y} ; t)$.

For open ASEP we write the corresponding probability as $P_{n}(\mathbf{x} ; t)$ (and do not specify any initial condition). The equation for $P_{n}$ is

$$
\begin{gathered}
\frac{d P_{n}}{d t}\left(x_{1}, \ldots, x_{n}: t\right)=\mathcal{Q}_{n} P_{n}\left(x_{1}, \ldots, x_{n}: t\right) \\
+\alpha \delta\left(x_{1}\right) P_{n-1}\left(x_{2}, \ldots, x_{n}: t\right)-\alpha\left(1-\delta\left(x_{1}\right)\right) P_{n}\left(x_{1}, \ldots, x_{n}: t\right) \\
-\beta \delta\left(x_{1}\right) P_{n}\left(x_{1}, \ldots, x_{n}: t\right)+\beta\left(1-\delta\left(x_{1}\right)\right) P_{n+1}\left(0, x_{1}, \ldots, x_{n}: t\right) .
\end{gathered}
$$

Define $\Phi_{n}(\mathbf{x} ; t)=e^{\alpha t} P_{n}(\mathbf{x} ; t)$. In terms of the operators $A_{n}$ and $B_{n}$ defined in (2) and (3), and $\delta=\delta\left(x_{1}\right)$, the equation for $\Phi_{n}$ becomes

$$
\frac{d \Phi_{n}(t)}{d t}=\mathcal{Q}_{n} \Phi_{n}+(\alpha-\beta) \delta \Phi_{n}+\alpha A_{n} \Phi_{n-1}+\beta B_{n} \Phi_{n+1}
$$

The equation and initial condition are satisfied if

$\Phi_{n}(t)=\int_{0}^{t} e^{(t-u) \mathcal{Q}_{n}}\left((\alpha-\beta) \delta \Phi_{n}(u)+\alpha A_{n} \Phi_{n-1}(u)+\beta B_{n} \Phi_{n+1}(u)\right) d u+e^{t \mathcal{Q}_{n}} \Phi_{n}(0)$.

We use the fact that the Laplace transform of a convolution is the product of the Laplace transforms. Recall that the kernel of $L_{n}(s)$ is $\widehat{\mathfrak{p}}(\mathbf{x}, \mathbf{y} ; s)$, which is the Laplace transform of the kernel of $e^{t \mathcal{Q}_{n}}$. In other words, the Laplace transform of the operator $e^{t \mathcal{Q}_{n}}$ is $L_{n}(s)$. So taking Laplace transforms in the last equation gives

$$
\widehat{\Phi}_{n}(s)=L_{n}(s)\left((\alpha-\beta) \delta \widehat{\Phi}_{n}(s)+\alpha A_{n} \widehat{\Phi}_{n-1}(s)+\beta B_{n} \widehat{\Phi}_{n+1}(s)\right)+L_{n}(s) \Phi_{n}(0) .
$$

If we now introduce the vector functions

$$
\widehat{\Phi}(s)=\left(\widehat{\Phi}_{n}(s)\right)_{n \geq 0}, \quad \Phi(0)=\left(\Phi_{n}(0)\right)_{n \geq 0},
$$

and the operator matrices $\delta, L(s), A, B$ defined earlier we see that the system may be written as

$$
\widehat{\Phi}(s)=L(s)((\alpha-\beta) \delta+\alpha A+\beta B) \widehat{\Phi}(s)+L(s) \Phi(0) .
$$

Since $\widehat{\Phi}(s)=\widehat{P}(s-\alpha)$, this gives the statement of Theorem 1 .

Corollaries 1.1 and 1.2: We write the operator inverse in (4) as

$$
(I-(M(s) L(s)(\alpha A+\beta B)))^{-1} M(s) L(s),
$$


where $M(s)$ is the diagonal matrix with operator entries $M_{n}(s)$. When $\beta=0$ this equals

$$
(I-\alpha M(s) L(s) A)^{-1} M(s) L(s) .
$$

The operator matrix $M(s) L(s) A$ consists of one subdiagonal, with $m, m-1$-entry $M_{m}(s) L_{m}(s) A_{m}$. Therefore the $n, n^{\prime}$-entry of the inverse $\left(n^{\prime} \leq n\right)$ is

$$
\alpha^{n-n^{\prime}} M_{n}(s) L_{n}(s) A_{n} \cdots M_{n^{\prime}+1}(s) L_{n^{\prime}+1}(s) A_{n^{\prime}+1},
$$

where for $n=n^{\prime}$ this equals $I$. So the $n, n^{\prime}$-entry of (77) is

$$
\alpha^{n-n^{\prime}} M_{n}(s) L_{n}(s) A_{n} \cdots M_{n^{\prime}+1}(s) L_{n^{\prime}+1}(s) A_{n^{\prime}+1} M_{n^{\prime}}(s) L_{n^{\prime}}(s) .
$$

Thus,

$\widehat{P}_{n}(s-\alpha)=\sum_{n^{\prime} \leq n} \alpha^{n-n^{\prime}} M_{n}(s) L_{n}(s) A_{n} \cdots M_{n^{\prime}+1}(s) L_{n^{\prime}+1}(s) A_{n^{\prime}+1} M_{n^{\prime}}(s) L_{n^{\prime}}(s) P_{n^{\prime}}(0)$

When $\alpha=0$ the matrix $M(s) L(s) B$ consists of one superdiagonal, and we obtain similarly

$$
\widehat{P}_{n}(s)=\sum_{n^{\prime} \geq n} \beta^{n^{\prime}-n} M_{n}(s) L_{n}(s) B_{n} \cdots M_{n^{\prime}-1}(s) L_{n^{\prime}-1}(s) B_{n^{\prime}-1} M_{n^{\prime}}(s) L_{n^{\prime}}(s) P_{n^{\prime}}(0) .
$$

If initially there are $k$ particles at $\mathbf{y} \in \mathcal{X}_{k}$ then in both cases $P_{n^{\prime}}(0)$ is nonzero only for $n^{\prime}=k$. The formulas become

$$
\begin{gathered}
\widehat{P}_{n}(s-\alpha)=\alpha^{n-k} M_{n}(s) L_{n}(s) A_{n} \cdots M_{k+1}(s) L_{k+1}(s) A_{k+1} M_{k}(s) L_{k}(s) \delta_{y} \\
\widehat{P}_{n}(s)=\beta^{k-n} M_{n}(s) L_{n}(s) B_{n} \cdots M_{k-1}(s) L_{k-1}(s) B_{k-1} M_{k}(s) L_{k}(s) \delta_{y},
\end{gathered}
$$

and the corollaries follow.

Remark 1.1: For (a) we use the fact that because $A_{n}$ has the factor $\delta$,

$$
M_{n}(s) L_{n}(s) A_{n}=\left(I-\alpha L_{n}(s) \delta\right)^{-1} L_{n}(s) \delta A_{n}=L_{n}(s) \delta\left(I-\alpha \delta L_{n}(s) \delta\right)^{-1} A_{n} .
$$

Since $\left(A_{n} f\right)\left(x_{1}, \mathbf{x}\right)=\delta\left(x_{1}\right)\left(R_{n-1} f\right)(\mathbf{x})$ and $\mathbf{x} \in \mathcal{E}_{n-1}^{+}$, we obtain statement (a) in different notation. For (b) we use

$$
\begin{gathered}
M_{k}(s)=\left(I-(\alpha-\beta) L_{k}(s) \delta\right)^{-1}=I+(\alpha-\beta) L_{k}(s) \delta\left(I-(\alpha-\beta) L_{k}(s) \delta\right)^{-1} \\
=I+(\alpha-\beta) L_{k}(s) \delta\left(I-(\alpha-\beta) \delta L_{k}(s) \delta\right)^{-1} .
\end{gathered}
$$


Thus,

$$
M_{k}(s) L_{k}(s)=L_{k}(s)+(\alpha-\beta) L_{k}(s) \delta\left(I-(\alpha-\beta) \delta L_{k}(s) \delta\right)^{-1} \delta L_{k}(s),
$$

and statement (b) follows.

Initially a single particle: Let $\mathcal{P}_{1}(y ; t)=\sum_{x \geq 0} P_{1}(x, y ; t)$ denote the probability that, starting with one particle at $y$, we still have one particle at time $t$. Denote its Laplace transform by $\widehat{\mathcal{P}}_{1}(y ; s)$.

We begin with the case $\beta=0$, so $\mathcal{P}_{1}(y ; t)$ is the probability that no new particle has been injected by time $t$.

By Corollary 1.1 the Laplace transform $\widehat{P}_{1}(x, y ; s-\alpha)$ is equal to $\left(M_{1}(s) L_{1}(s) \delta_{y}\right)(x)$. By Remark 1.1(b) this equals

$$
\widehat{\mathfrak{p}}(x, y ; s)+\alpha \widehat{\mathfrak{p}}(x, 0 ; s)(1-\alpha \widehat{\mathfrak{p}}(0,0 ; s))^{-1} \widehat{\mathfrak{p}}(0, y ; s) .
$$

Then using $\sum_{x \geq 0} \widehat{\mathfrak{p}}(x, y ; s)=s^{-1}$ we obtain

$$
\widehat{\mathcal{P}}_{1}(y ; s-\alpha)=\frac{1}{s}\left[1+\frac{\alpha \widehat{\mathfrak{p}}(0, y ; s)}{1-\alpha \widehat{\mathfrak{p}}(0,0 ; s)}\right] .
$$

From formula (23) for $\mathfrak{p}(x, y ; t)$ in the case $n=1$ we compute that

$$
\widehat{\mathfrak{p}}(0, y ; s)=\frac{1}{q} \frac{\xi_{+}(s)^{-y}}{\xi_{+}(s)-1},
$$

where

$$
\xi_{+}(s)=\frac{1}{2 q}\left(s+1+\sqrt{(s+1)^{2}-4 p q}\right) ;
$$

this is the solution of $\varepsilon(\xi)=s$ with positive square root when $s>0$. Thus

$$
\widehat{\mathcal{P}}_{1}(y ; s-\alpha)=\frac{1}{s}\left[1+\frac{\alpha \xi_{+}(s)^{-y}}{q\left(\xi_{+}(s)-1\right)-\alpha}\right] .
$$

The denominator in the brackets is nonzero for $s=\alpha$ (and positive for $s>\alpha$ ), from which we conclude that

$$
\int_{0}^{\infty} \mathcal{P}_{1}(y ; t) d t=\widehat{\mathcal{P}}_{1}(y ; 0)=\frac{1}{\alpha}+\frac{\xi_{+}(\alpha)^{-y}}{q\left(\xi_{+}(\alpha)-1\right)-\alpha} .
$$

It follows that with probability one a second particle will eventually be injected, since $\mathcal{P}(y ; t) \rightarrow 0$ as $t \rightarrow \infty$, and the integral is the expected time at which it occurs 9

\footnotetext{
${ }^{9}$ If $T(y)$ denotes the time when a second particle is injected, then $\operatorname{Prob}(T(y)>t)=\mathcal{P}_{1}(y ; t)$, from which the statement follows.
} 
This was for $\beta=0$. For $\alpha=0, \mathcal{P}_{1}(y ; t)$ is the probability that the particle has not been ejected by time $t$. We use Corollary 1.2 (and Remark 1.1), and formula (11) is replaced by

$$
\widehat{\mathcal{P}}_{1}(y ; s)=\frac{1}{s}\left[1-\frac{\beta \xi_{+}(s)^{-y}}{q\left(\xi_{+}(s)-1\right)+\beta}\right] \text {. }
$$

We compute that as $s \rightarrow 0$,

$$
\begin{gathered}
\widehat{\mathcal{P}}_{1}(y ; s) \sim \frac{1}{s}\left[1-\frac{\beta\left(q^{-1}-1\right)^{-y}}{p-q+\beta}\right] \quad \text { if } p>q, \\
\widehat{\mathcal{P}}_{1}(y ; s) \sim \frac{1}{\sqrt{2 s}}(2 y+1 / \beta) \text { if } p=q, \\
\widehat{\mathcal{P}}_{1}(y ; s) \rightarrow \frac{y+q / \beta}{q-p} \text { if } p<q .
\end{gathered}
$$

Applying the Tauberian theorem we deduce

$$
\begin{gathered}
\lim _{t \rightarrow \infty} \mathcal{P}_{1}(y ; t)=1-\frac{\beta\left(q^{-1}-1\right)^{-y}}{p-q+\beta} \text { if } p>q, \\
\mathcal{P}_{1}(y ; t) \sim \frac{1}{\sqrt{2 \pi}}\left[2 y+\frac{1}{\beta}\right] t^{-1 / 2} \text { as } t \rightarrow \infty \text { if } p=q, \\
\int_{0}^{\infty} \mathcal{P}_{1}(y ; t) d t=\frac{y+q / \beta}{q-p} \text { if } p<q .
\end{gathered}
$$

When $p>q$ the limit on the first line is the probability that the particle is never ejected. If $p \leq q$, then with probability 1 the particle will eventually be ejected. The expected value of the time at which it is ejected is infinite when $p=q$, by the second line, and is given by the integral on the last line when $p<q$.

\section{B. TASEP}

Remark 1.2: To compute $\left(I-\alpha L_{n}^{0}(s)\right)^{-1}$ we use the fact that for $k \geq 1$ the kernel of $L_{n}^{0}(s)^{k}$ is

$$
\sum_{\mathbf{z}_{1}, \ldots, \mathbf{z}_{k-1} \in \mathcal{X}_{n}^{+}} \widehat{\mathfrak{p}}\left((0, \mathbf{x}),\left(0, \mathbf{z}_{1}\right) ; s\right) \widehat{\mathfrak{p}}\left(\left(0, \mathbf{z}_{1}\right),\left(0, \mathbf{z}_{2}\right) ; s\right) \cdots \widehat{\mathfrak{p}}\left(\left(0, \mathbf{z}_{k-1}\right),(0, \mathbf{y}) ; s\right) .
$$

The summand is the Laplace transform of the $(k-1)$-fold convolution

$$
\int_{u_{1}+\cdots+u_{k-1}=t} \mathfrak{p}\left((0, \mathbf{x}),\left(0, \mathbf{z}_{1}\right) ; u_{1}\right) \mathfrak{p}\left(\left(0, \mathbf{z}_{1}\right),\left(0, \mathbf{z}_{2}\right) ; u_{2}\right) \cdots \mathfrak{p}\left(\left(0, \mathbf{z}_{n-1}\right),(0, \mathbf{y}) ; u_{k-1}\right) d \mathbf{u}
$$


When $p=1$, if the left-most particle begins at 0 and ends at 0 then it was always at 0 . It follows that these probabilities have the semigroup property. Thus after summing over $\mathbf{z}_{1}, \ldots, \mathbf{z}_{k-1}$ the integral becomes

$$
\int_{u_{1}+\cdots+u_{k-1}=t} \mathfrak{p}((0, \mathbf{x}),(0, \mathbf{y}), t) d \mathbf{u}=\frac{t^{k-1}}{(k-1) !} \mathfrak{p}((0, \mathbf{x}),(0, \mathbf{y}), t) .
$$

Therefore the kernel of of $L_{n}^{0}(s)^{k}$ is

$$
\int_{0}^{\infty} e^{-s t} \frac{t^{k-1}}{(k-1) !} \mathfrak{p}((0, \mathbf{x}),(0, \mathbf{y}), t) d t
$$

It follows that the kernel of $\sum_{k=1}^{\infty}\left(\alpha L_{n}^{0}(s)\right)^{k}$ is

$$
\alpha \int_{0}^{\infty} e^{-s t} e^{\alpha t} \mathfrak{p}((0, \mathbf{x}),(0, \mathbf{y}), t) d t=\alpha \widehat{\mathfrak{p}}((0, \mathbf{x}),(0, \mathbf{y}) ; s-\alpha)
$$

This gives $\left(I-\alpha L_{n}^{0}(s)\right)^{-1}=1+\alpha L_{n}^{0}(s-\alpha)$.

Once we have this result (and Corollary 1.2, Remark 1.1, and the determinant formula (24) for $\mathfrak{p}(\mathbf{x}, \mathbf{y} ; t)$ ), we have all the ingredients necessary to compute $\mathcal{P}_{n}(t)$ for small $n$. This is the probability that starting with no particles at time 0 there are exactly $n$ particles at time $t$, which equals $\sum_{\mathbf{x} \in \mathcal{X}_{n}} \mathcal{P}_{n}(\mathbf{x} ; t)$. The computation of products of kernels involves summing geometric series.

\section{SSEP}

Proof of Theorem 2: A state of the system is a function $\eta: \mathbb{Z}^{+} \rightarrow\{0,1\}$ where $\eta_{x}=1$ means site $x$ is occupied and $\eta_{x}=0$ means site $x$ is not occupied. Recall that we defined $\Psi_{n}\left(x_{1}, x_{2}, \ldots, x_{n} ; t\right)$ as the probability that sites $x_{1}, \ldots, x_{n}$ are occupied at time $t$. Thus,

$$
\Psi_{n}\left(x_{1}, x_{2}, \ldots, x_{n} ; t\right)=\left\langle\eta_{x_{1}}(t) \cdots \eta_{x_{n}}(t)\right\rangle
$$

The Markov generator $\mathcal{L}$ of ASEP on $\mathbb{Z}^{+}$with an open boundary at zero is given [4] by

$$
\begin{aligned}
& \mathcal{L} f(\eta)=\alpha\left(1-\eta_{0}\right)\left(f\left(\eta^{0}\right)-f(\eta)\right)+\beta \eta_{0}\left(f\left(\eta^{0}\right)-f(\eta)\right) \\
& +\sum_{k=0}^{\infty}\left[p \eta_{k}\left(1-\eta_{k+1}\right)+q\left(1-\eta_{k}\right) \eta_{k+1}\right]\left(f\left(\eta^{k, k+1}\right)-f(\eta)\right) .
\end{aligned}
$$

Here $f$ is an $\mathbb{R}$-valued function that depends on only finitely many sites, and

$$
\left(\eta^{k}\right)_{x}=\left\{\begin{array}{ll}
1-\eta_{x} & \text { if } x=k \\
\eta_{x} & \text { if } x \neq k,
\end{array} \quad\left(\eta^{k, k+1}\right)_{x}= \begin{cases}\eta_{k+1} & \text { if } x=k \\
\eta_{k} & \text { if } x=k+1 \\
\eta_{x} & \text { if } x \neq k, k+1\end{cases}\right.
$$


For SSEP the sum in (12) equals $1 / 2$ times

$$
\sum_{k=0}^{\infty}\left[\eta_{k}\left(1-\eta_{k+1}\right)+\left(1-\eta_{k}\right) \eta_{k+1}\right]\left(f\left(\eta^{k, k+1}\right)-f(\eta)\right) .
$$

When $\eta_{k}=\eta_{k+1}$ the first factor equals 0 ; otherwise it equals 1 . Since the second factor is zero when $\eta_{k}=\eta_{k+1}$, we can ignore the first factor, and we get

$$
\sum_{k=0}^{\infty}\left(f\left(\eta^{k, k+1}\right)-f(\eta)\right)
$$

For the correlations we are interested in $f(\eta)=\eta_{x_{1}} \cdots \eta_{x_{n}}$, so the $k$ th summand equals zero unless either $k=x_{i}$ for some $i$ or $k=x_{i}-1$ for some $i$. (We'll see that these cannot both happen for a nonzero summand.)

Suppose first that $k=x_{i}$. If $x_{i+1}=x_{i}+1$ then $k+1=x_{i+1}$ and the substitution $\eta \rightarrow \eta^{k, k+1}$ applied to $f(\eta)$ just interchanges $\eta_{x_{i}}$ and $\eta_{x_{i+1}}$. Therefore the $k$ th summand is zero. It follows that for a nonzero summand we must have $x_{i+1}>x_{i}+1=k+1$, and the substitution $\eta \rightarrow \eta^{k, k+1}$ only affects the $i$ th factor in $f(\eta)$. Therefore the summand equals

$$
\left(\eta_{x_{1}} \cdots \eta_{x_{i-1}} \eta_{x_{i}+1} \eta_{x_{i+1}} \cdots \eta_{x_{n}}-\eta_{x_{1}} \cdots \eta_{x_{i-1}} \eta_{x_{i}} \eta_{x_{i+1}} \cdots \eta_{x_{n}}\right)\left(1-\delta\left(x_{i+1}-x_{i}-1\right)\right)
$$

Similarly if $k=x_{i}-1$ the summand equals

$$
\left(\eta_{x_{1}} \cdots \eta_{x_{i-1}} \eta_{x_{i}-1} \eta_{x_{i+1}} \cdots \eta_{x_{n}}-\eta_{x_{1}} \cdots \eta_{x_{i-1}} \eta_{x_{i}} \eta_{x_{i+1}} \cdots \eta_{x_{n}}\right)\left(1-\delta\left(x_{i}-x_{i-1}-1\right)\right)
$$

(This is to be multiplied by $1-\delta\left(x_{1}\right)$ when $i=1$.)

If $k=x_{i}$ for (13) and $k=x_{i^{\prime}}-1$ for (14) then $i^{\prime}=i+1$, so $x_{i+1}=x_{i^{\prime}}=x_{i}+1$ and (13) zero, and $x_{i^{\prime}}=x_{i}+1=x_{i^{\prime}-1}+1$ so (14) is zero. Thus for the $k$ th summand to be nonzero either $k=x_{i}$ for some $i$ or $k=x_{i}-1$ for some $i$, but not both.

It follows that for SSEP the expected value of the sum in (12) is equal to $1 / 2$ times the sum over $i$ of the expected values of the sum of (13) and (14). This equals $\mathcal{Q}_{n}\left(\left\langle\eta_{x_{1}} \cdots \eta_{x_{n}}\right\rangle\right)$

Adding what we get from the first two terms of (12) we find that the differential equation for $\Psi_{n}=\Psi_{n}(t)$ is

$$
\begin{gathered}
\frac{d}{d t} \Psi_{n}\left(x_{1}, x_{2}, \ldots, x_{n}\right)=\mathcal{Q}_{n} \Psi_{n}\left(x_{1}, x_{2}, \ldots, x_{n}\right) \\
-(\alpha+\beta) \Psi_{n}\left(x_{1}, x_{2}, \ldots, x_{n}\right) \delta\left(x_{1}\right)+\alpha \Psi_{n-1}\left(x_{2}, \ldots, x_{n}\right) \delta\left(x_{1}\right) 10
\end{gathered}
$$

\footnotetext{
${ }^{10}$ Observe that the constant functions $\Psi_{n}=(\alpha /(\alpha+\beta))^{n}$ satisfy the equations. Thus the Bernoulli measure with density $\rho=\alpha /(\alpha+\beta)$ is stationary. This also follows from the results of 4 in which stationary measures were determined for general ASEP on $\mathbb{Z}^{+}$.
} 
Except for the change $\alpha-\beta \rightarrow-(\alpha+\beta)$, this is (6) without the $B_{n}$ terms. Therefore to complete the proof of Theorem 2 we need only make this change as we go through the rest of the proof of Theorem 1 , which we need not do.

Corollaries 2.1 and 2.2: Just as (8) is obtained from Theorem 1, we obtain now for $n>0$,

$$
\widehat{\Psi}_{n}(s)=\sum_{k=0}^{n} \alpha^{n-k} M_{n}(s) L_{n}(s) A_{n} \cdots M_{k+1}(s) L_{k+1}(s) A_{k+1} M_{k}(s) L_{k}(s) \Psi_{k}(0) .
$$

Corollary 2.1 follows. For Corollary 2.2 we have $\Psi_{n}(0)=\rho^{n}$, and we use the fact that $L_{n}(1)=\sum_{\mathbf{y} \in \mathcal{X}_{n}} \widehat{\mathfrak{p}}(\mathbf{x}, \mathbf{y} ; s)=s^{-1}$. Corollary 2.3 is the case $\rho=0$ of Corollary 2.2.

$\Delta N(t)$, the net number of particles that entered the system: We assume that we have SSEP with Bernoulli initial condition. Corollary 2.2 when $n=1$ gives, for the Laplace transform of $\left\langle\eta_{x}(t)\right\rangle$,

$$
\widehat{\left\langle\eta_{x}\right\rangle}(s)=\widehat{\Psi}_{1}(x ; s)=s^{-1}\left(\rho M_{1}(s) 1+\alpha M_{1}(s) L_{1}(s) A_{1} 1\right) .
$$

By Remark 1.1(a) applied here, we have11

$$
M_{1}(s) L_{1}(s) A_{1} 1=\frac{\widehat{\mathfrak{p}}(x, 0 ; s)}{1+\gamma \widehat{\mathfrak{p}}(0,0 ; s)}
$$

where we set

$$
\gamma=\alpha+\beta
$$

Similarly, from (9) we obtain

$$
M_{1}(s) 1=1-\gamma \frac{\widehat{\mathfrak{p}}(x, 0 ; s)}{1+\gamma \widehat{\mathfrak{p}}(0,0 ; s)} .
$$

Combining the two gives

$$
\widehat{\left\langle\eta_{x}\right\rangle}(s)=\frac{1}{s}\left[\rho+\frac{\alpha-\gamma \rho}{1+\gamma \widehat{\mathfrak{p}}(0,0 ; s)} \widehat{\mathfrak{p}}(x, 0 ; s)\right] .
$$

Subtracting $\rho / s$ from both sides and summing over $x \geq 0$ we get for the Laplace transform of $\langle\Delta N(t)\rangle$

$$
\langle\widehat{\Delta N(t)}\rangle(s)=\frac{1}{s^{2}} \frac{\alpha-\gamma \rho}{1+\gamma \widehat{\mathfrak{p}}(0,0 ; s)} .
$$

\footnotetext{
${ }^{11}$ Since the definition of $M_{k}(s)$ is different now we must replace $\alpha-\beta$ by $-(\alpha+\beta)$ when using the remark.
} 
From (10) we have for SSEP

$$
\widehat{\mathfrak{p}}(0,0 ; s)=\frac{2}{s+\sqrt{s^{2}+2 s}} \sim \sqrt{\frac{2}{s}} \text { as } s \rightarrow 0 .
$$

Hence

$$
\langle\widehat{\Delta N(t)}\rangle(s) \sim \frac{\alpha-\gamma \rho}{\sqrt{2} \gamma} s^{-3 / 2} \text { as } s \rightarrow 0
$$

By the Tauberian theeorem this implies

$$
\langle\Delta N(t)\rangle \sim \sqrt{\frac{2}{\pi}} \frac{\alpha-\gamma \rho}{\gamma} t^{1 / 2} \text { as } t \rightarrow \infty .
$$

As stated in the last section the second moment $\left\langle\Delta N(t)^{2}\right\rangle$ is finite. To show this we use that the second moment is equal to

$$
\lim _{N \rightarrow \infty} \sum_{x_{1}, x_{2}<N}\left\langle\left(\eta_{x_{1}}(t)-\eta_{x_{1}}(0)\right)\left(\eta_{x_{2}}(t)-\eta_{x_{2}}(0)\right)\right\rangle .
$$

We can show that the sum is a polynomial of degree two in $\rho$, and that each of the three coefficients of the powers of $\rho$ has a limit as $N \rightarrow \infty$. The argument is quite involved, and we do not include it.

The second moment of $\Delta N(t)$ when $\rho=0$ : Now it is mainly a question of determining the asymptotics of $\sum_{x_{1}<x_{2}}\left\langle\eta_{x_{1}} \eta_{x_{2}}\right\rangle$. The Laplace transform of $\Psi_{2}(\mathbf{x} ; t)=$ $\left\langle\eta_{x_{1}} \eta_{x_{2}}\right\rangle$ is given by Corollary 2.2 as

$$
\widehat{\Psi}_{2}(\mathbf{x} ; s)=\alpha^{2} s^{-1} M_{2}(s) L_{2}(s) A_{2} M_{1}(s) L_{1}(s) A_{1} 1 .
$$

We computed $M_{1}(s) L_{1}(s) A_{1} 1$ in (15) . Combining this with Remark 1.1(a) gives

$$
\widehat{\Psi}_{2}(\mathbf{x} ; s)=\alpha^{2} s^{-1} L_{2,1}^{0}(s)\left(I+\gamma L_{1}^{0}(s)\right)^{-1} \frac{\widehat{\mathfrak{p}}_{0}}{1+\gamma \widehat{\mathfrak{p}}_{0}(0)},
$$

where $\widehat{\mathfrak{p}}_{0}$ is the function $x \rightarrow \widehat{\mathfrak{p}}(x, 0 ; s)$.

To obtain the Laplace transform $\sum_{x_{1}<x_{2}}\left\langle\widehat{\eta_{x_{1}} \eta_{x_{2}}}\right\rangle$ we sum over $\mathbf{x} \in \mathcal{X}_{2}$. If we recall that the kernel of $L_{2,1}^{0}(s)$ is $\widehat{\mathfrak{p}}(\mathbf{x},(0, y))$ and that the sum of this over $\mathbf{x} \in \mathcal{X}_{2}$ is $s^{-1}$ we see that the desired sum is the inner product of the remaining function with the constant function $s^{-1}$. Thus,

$$
\sum_{x_{1}<x_{2}}\left\langle\widehat{\eta_{x_{1}} \eta_{x_{2}}}\right\rangle=\frac{\alpha^{2} s^{-2}}{1+\gamma \widehat{\mathfrak{p}}_{0}(0)}\left(\left(I+\gamma L_{1}^{0}(s)\right)^{-1} \widehat{\mathfrak{p}}_{0}, 1\right) .
$$


(Here we use the fact that $\widehat{\mathfrak{p}}_{0}$ belongs to $L^{1}$ and that $L_{1}^{0}(s)$ is a bounded operator on this space.)

What follows is not rigorous. We want to rescale $\left(I+\gamma L_{1}^{0}(s)\right)^{-1}$ as $s \rightarrow 0$, and we refer to formula (23) given in the Appendix for $\mathfrak{p}(\mathbf{x}, \mathbf{y} ; t)$. After taking Laplace transforms in the case $n=2$ we find that $L_{1}^{0}(s)$ has kernel

$$
L_{1}^{0}(x, y ; s)=\sum_{\sigma \in \mathbb{B}_{2}} \frac{1}{(2 \pi i)^{2}} \int_{\mathcal{C}_{R}} \int_{\mathcal{C}_{R}} A_{\sigma} \frac{\xi_{\sigma(2)}^{x} \xi_{2}^{-y}}{s-\varepsilon\left(\xi_{1}\right)-\varepsilon\left(\xi_{2}\right)} \frac{d \xi_{1} d \xi_{2}}{\xi_{1} \xi_{2}}
$$

where $\mathcal{C}_{R}$ is a circle with radius $R$ with $R$ large. (Some integrals are taken over two pairs of different contours and the results averaged.) To begin with, $s$ is so large that taking Laplace transforms under the integral sign is valid.

If we ignore the poles of the $A_{\sigma}$ we can move both contours to the unit circle $\mathcal{C}_{1}$. Then the range of $\varepsilon\left(\xi_{1}\right)+\varepsilon\left(\xi_{2}\right)$ is $[-4,0]$, so we may take any $s>0$. As $s \rightarrow 0$ the main contribution comes from a neighborhood of $\xi_{1}=\xi_{2}=1$ because the denominator vanishes there when $s=0$. If we set $\xi_{1}=e^{i v_{1}}, \xi_{2}=e^{i v_{2}}$ then the integral with its factor becomes to first order

$$
\begin{gathered}
\frac{1}{4 \pi^{2}} \int_{\mathbb{R}} \int_{\mathbb{R}} A_{\sigma}\left(e^{i v_{1}}, e^{i v_{2}}\right) \frac{e^{i\left(v_{\sigma(2)} x-v_{2} y\right)}}{s+\left(v_{1}^{2}+v_{2}^{2}\right) / 2} d v_{1} d v_{2} \\
=\frac{1}{2 \pi^{2}} \int_{\mathbb{R}} \int_{\mathbb{R}} A_{\sigma}\left(e^{i \sqrt{2 s} v_{1}}, e^{i \sqrt{2 s} v_{2}}\right) \frac{e^{i \sqrt{2 s}\left(v_{\sigma(2)} x-v_{2} y\right)}}{1+v_{1}^{2}+v_{2}^{2}} d v_{1} d v_{2} .
\end{gathered}
$$

This becomes, after the scaling $x \rightarrow x / \sqrt{2 s}, y \rightarrow y / \sqrt{2 s}$,

$$
\frac{1}{2 \pi^{2} \sqrt{2 s}} \int_{\mathbb{R}} \int_{\mathbb{R}} A_{\sigma}\left(e^{i \sqrt{2 s} v_{1}}, e^{i \sqrt{2 s} v_{2}}\right) \frac{e^{i\left(v_{\sigma(2)} x-v_{2} y\right)}}{1+v_{1}^{2}+v_{2}^{2}} d v_{1} d v_{2}
$$

which acts on functions on $\mathbb{R}^{+}$.

Each $A_{\sigma}$ has absolute value 1 on $\mathcal{C}_{1} \times \mathcal{C}_{1}$, and each $A_{\sigma}\left(e^{i \sqrt{2 s} v_{1}}, e^{i \sqrt{2 s} v_{2}}\right)$ has limit 1 as $s \rightarrow 0$ except when $v_{1}=v_{2}(\bmod 2 \pi)$. Thus we replace the above by the approximation

$$
\frac{1}{2 \pi^{2} \sqrt{2 s}} \int_{\mathbb{R}} \int_{\mathbb{R}} \frac{e^{i\left(v_{\sigma(2)} x-v_{2} y\right)}}{1+v_{1}^{2}+v_{2}^{2}} d v_{1} d v_{2}
$$

This depends only on $\sigma(2)$. If we use the fact that the denominator is even in each $v_{i}$, and that $v_{-i}=-v_{i}$, we see that the sum over $\sigma \in \mathbb{B}_{2}$ of the integrals equals

$$
\frac{1}{\pi^{2} \sqrt{2 s}} \int_{\mathbb{R}} \int_{\mathbb{R}} \frac{e^{i v_{2}(x-y)}+e^{i v_{2}(x+y)}}{1+v_{1}^{2}+v_{2}^{2}} d v_{1} d v_{2}+\frac{2}{\pi^{2} \sqrt{2 s}} \int_{\mathbb{R}} \int_{\mathbb{R}} \frac{e^{i\left(v_{1} x-v_{2} y\right)}}{1+v_{1}^{2}+v_{2}^{2}} d v_{1} d v_{2} .
$$


In the first integral we integrate first with respect to $v_{1}$, and we obtain

$$
\frac{1}{\pi \sqrt{2 s}} \int_{\mathbb{R}} \frac{e^{i v(x-y)}+e^{i v(x+y)}}{\sqrt{1+v^{2}}} d v+\frac{2}{\pi^{2} \sqrt{2 s}} \int_{\mathbb{R}} \int_{\mathbb{R}} \frac{e^{i\left(v_{1} x-v_{2} y\right)}}{1+v_{1}^{2}+v_{2}^{2}} d v_{1} d v_{2}{ }^{12}
$$

These are of the order $1 / \sqrt{s}$ as $s \rightarrow 0$. Now we indicate why the contributions from the poles of the $A_{\sigma}$, when we shrink the contours, are of lower order.

Consider the permutations $( \pm 21)$, when $A_{\sigma}=S\left(\xi_{2}, \xi_{1}\right)$. (Times $\xi_{1}^{-1}$ when $\sigma=$ $(-21)$; this has no effect on what follows.) With the $\xi_{2}$-integration over $\mathcal{C}_{R}$, we shrink the $\xi_{1}$-contour to $\mathcal{C}_{1}$. Then when we shrink the $\xi_{2}$-contour we pass the pole at $\xi_{2}=2-\xi_{1}^{-1}$ for all $\xi_{1} \in \mathcal{C}_{1}$. The residue is a constant times

$$
\int_{\mathcal{C}_{1}}\left(\frac{\xi-1}{\xi}\right)^{2} \frac{\xi^{x}\left(2-\xi^{-1}\right)^{-y-1}}{s-\frac{(\xi-1)^{2}}{2 \xi-1}} \frac{d \xi}{\xi}
$$

where we replaced $\xi_{1}$ by $\xi$. With either branch of $\xi^{1 / 2}$ we may write

$$
\frac{(\xi-1)^{2}}{2 \xi-1}=\frac{\left(\xi^{1 / 2}-\xi^{-1 / 2}\right)^{2}}{2-\xi^{-1}}
$$

On $\mathcal{C}_{1}$ the numerator is negative real (except when $\xi=1$ ) while the denominator lies in the right half-plane. Thus the quotient lies in the left half-plane (except when $\xi=1$ ) so we may take any $s>0$ in the integral. Since again the main contribution comes from near $\xi=1$, we set $\xi=e^{i v}$ and make the replacements $2-\xi^{-1} \rightarrow 2-(1-i v)=1+i v \rightarrow e^{i v}$, and we get

$$
\int_{\mathbb{R}} \frac{v^{2}}{s+v^{2}} e^{i v(x-y)} d v=\sqrt{s} \int_{\mathbb{R}} \frac{v^{2}}{1+v^{2}} e^{i \sqrt{s} v(x-y)} d v .
$$

After the scaling this becomes independent of $s$.

The factor $(\xi-1)^{2}$ in the integrand in (19) was important. It also appears in the residues for the other integrals, which also become independent of $s$ by similar computations. We omit the details.

Thus when we scale the contributions from the poles of the $A_{\sigma}$ are independent of $s$, and so of lower order than the main terms (18).

Set

$$
\begin{aligned}
& J_{1}(x, y)=\frac{1}{2 \pi} \int_{\mathbb{R}} \frac{e^{i v(x-y)}+e^{i v(x+y)}}{\sqrt{1+v^{2}}} d v, \\
& J_{2}(x, y)=\frac{1}{\pi^{2}} \int_{\mathbb{R}} \int_{\mathbb{R}} \frac{e^{i\left(v_{1} x-v_{2} y\right)}}{1+v_{1}^{2}+v_{2}^{2}} d v_{1} d v_{2} .
\end{aligned}
$$

\footnotetext{
${ }^{12}$ The integrals are $K_{0}$ Bessel functions, but this fact is not useful.
} 
We showed that $1+\gamma L_{1}^{0}(s)$, when scaled, is equal to $2 \gamma / \sqrt{2 s}\left(J_{1}+J_{2}\right)$ plus an operator independent of $s$. Therefore we presume that to a first approximation the scaled operator $\left(1+\gamma L_{1}^{0}(s)\right)^{-1}$ is equal to $(2 \gamma)^{-1} \sqrt{2 s}\left(J_{1}+J_{2}\right)^{-1}$. Also, we see from (10) and the symmetry of $\widehat{\mathfrak{p}}(\mathbf{x}, \mathbf{y} ; s)$ that

$$
\widehat{\mathfrak{p}}_{0}(x)=\widehat{\mathfrak{p}}(x, 0 ; s) \sim \sqrt{\frac{2}{s}} e^{-\sqrt{2 s} x} \quad \text { as } s \rightarrow 0 .
$$

This gives the Conjecture

$$
\left(\left(I+\gamma L_{1}^{0}(s)\right)^{-1} \widehat{\mathfrak{p}}_{0}, 1\right) \sim \frac{1}{\gamma \sqrt{2 s}}\left(\left(J_{1}+J_{2}\right)^{-1} e^{-x}, 1\right) \quad \text { as } s \rightarrow 0 .
$$

We shall show that the inner product equals $2 / \pi$. Assume this, and the conjecture, for now. Then from (16) and the asymptotics $\widehat{\mathfrak{p}}(0,0 ; s) \sim \sqrt{2 / s}$ as $s \rightarrow 0$ we find that

$$
\sum_{x_{1}<x_{2}}\left\langle\widehat{\eta_{x_{1}} \eta_{x_{2}}}\right\rangle \sim \frac{1}{\pi} \frac{\alpha^{2}}{\gamma^{2}} s^{-2} \text { as } s \rightarrow 0
$$

Therefore by the Tauberian theorem,

$$
\sum_{x_{1}<x_{2}}\left\langle\eta_{x_{1}} \eta_{x_{2}}\right\rangle \sim \frac{1}{\pi} \frac{\alpha^{2}}{\gamma^{2}} t \quad \text { as } t \rightarrow \infty
$$

Since

$$
\left\langle\Delta N(t)^{2}\right\rangle=\sum_{x_{1}, x_{2} \geq 0}\left\langle\eta_{x_{1}} \eta_{x_{2}}\right\rangle=2 \sum_{x_{1}<x_{2}}\left\langle\eta_{x_{1}} \eta_{x_{2}}\right\rangle+\langle\Delta N(t)\rangle
$$

and $\Delta N(t)=o(t)$, we get the result stated in Section II,

$$
\left\langle\Delta N(t)^{2}\right\rangle \sim \frac{2}{\pi} \frac{\alpha^{2}}{\gamma^{2}} t \quad \text { as } t \rightarrow \infty
$$

Now we show that the inner product in (20) equals $2 / \pi$. We first use an observation about Wiener-Hopf plus Hankel operators, of which $J_{1}$ is one. (Recall that our operators act on functions on $\mathbb{R}^{+}$.) Suppose we have such an operator with kernel $J(x-y)+J(x+y)$, so that the result of its action on a function $f$ on $\mathbb{R}^{+}$is

$$
(J f)(x)=\int_{0}^{\infty}(J(x-y)+J(x+y)) f(y) d y .
$$

If we extend $f$ to an even function on $\mathbb{R}$, then this equals

$$
\int_{-\infty}^{\infty} J(x-y) f(y) d y .
$$


So the operator becomes simply convolution by $J$.

Also, it follows from the fact that $J_{2}(x, y)$ is even in $y$ that if $f$, defined on $\mathbb{R}^{+}$, is extended to be even on $\mathbb{R}$ then

$$
\left(J_{2} f\right)(x)=\frac{1}{2} \int_{-\infty}^{\infty} J_{2}(x, y) f(y) d y .
$$

To recapitulate, define

$$
\begin{gathered}
K_{1}(x, y)=\frac{1}{2 \pi} \int_{\mathbb{R}} \frac{e^{i v(x-y)}}{\sqrt{1+v^{2}}} d v, \\
K_{2}(x, y)=\frac{1}{2 \pi^{2}} \int_{\mathbb{R}} \int_{\mathbb{R}} \frac{e^{i\left(v_{1} x-v_{2} y\right)}}{1+v_{1}^{2}+v_{2}^{2}} d v_{1} d v_{2},
\end{gathered}
$$

acting on functions on $\mathbb{R}$. Then on $\mathbb{R}^{+}$we have $\left(J_{1}+J_{2}\right) f=\left(K_{1}+K_{2}\right) f$, where the $f$ on the right is the even extension of the $f$ on the left.

It follows from this that for an even function $f$ on $\mathbb{R},\left(J_{1}+J_{2}\right)^{-1} f$ is the restriction to $\mathbb{R}^{+}$of $\left(K_{1}+K_{2}\right)^{-1} f 13$ Therefore the inner product in (20), which is over $\mathbb{R}^{+}$, is equal to

$$
\frac{1}{2}\left(\left(K_{1}+K_{2}\right)^{-1} e^{-|x|}, 1\right)
$$

where this inner product is over $\mathbb{R}$.

Because of the forms of the kernels of $K_{1}$ and $K_{2}$, the operators simplify when we conjugate with the Fourier transform. The operator $K_{1}$ becomes $\widehat{K}_{1}$, which is multiplication by the function $1 / \sqrt{1+v^{2}}$, and the operator $K_{2}$ becomes $\widehat{K}_{2}$, which has kernel

$$
\widehat{K}_{2}(u, v)=\frac{1}{\pi} \frac{1}{1+u^{2}+v^{2}}
$$

Since the Fourier transform of 1 with factor $1 / 2 \pi$ outside the integral is $\delta_{0}$ and the Fourier transform of $e^{-|x|}$ with factor 1 is $2 /\left(1+v^{2}\right)$, we see that (21) (which equals the inner product in (20) ) equals

$$
\left(\left(\widehat{K}_{1}+\widehat{K}_{2}\right)^{-1}\left(1+v^{2}\right)^{-1}, \delta_{0}\right)
$$

If we use

$$
\left(\widehat{K}_{1}+\widehat{K}_{2}\right)^{-1}=\widehat{K}_{1}^{-1 / 2}\left(1+\widehat{K}_{1}^{-1 / 2} \widehat{K}_{2} \widehat{K}_{1}^{-1 / 2}\right)^{-1} \widehat{K}_{1}^{-1 / 2}
$$

and the fact that $\widehat{K}_{1}^{-1 / 2} \delta_{0}=\delta_{0}$, we see that the above equals

$$
\left((I+\widehat{K})^{-1} \psi, \delta_{0}\right)
$$

\footnotetext{
${ }^{13}$ This uses that $K_{1}+K_{2}$ commutes with the operator $f(x) \rightarrow f(-x)$.
} 
where

$$
\widehat{K}(u, v)=\frac{1}{\pi} \frac{\left(1+u^{2}\right)^{1 / 4}\left(1+v^{2}\right)^{1 / 4}}{1+u^{2}+v^{2}}, \quad \psi(v)=\left(1+v^{2}\right)^{-3 / 4} .
$$

If we conjugate with the unitary operator $f(u) \rightarrow(\cosh x)^{1 / 2} f(\sinh x)$ we find that (22) equals $\left((I+\tilde{K})^{-1} \widetilde{\psi}, \delta_{0}\right)$, where

$$
\begin{gathered}
\tilde{K}(x, y)=(\cosh x)^{1 / 2} \widehat{K}(\sinh x, \sinh y)(\cosh y)^{1 / 2}=\frac{1}{\pi} \frac{\cosh x \cosh y}{1+\sinh ^{2} x+\sinh ^{2} y}, \\
\widetilde{\psi}(x)=(\cosh x)^{1 / 2} \psi(\sinh x)=\operatorname{sech} x .
\end{gathered}
$$

From

$$
\begin{aligned}
& \cosh x \cosh y=\frac{1}{2}(\cosh (x+y)+\cosh (x-y)), \\
& 1+\sinh ^{2} x+\sinh ^{2} y=\cosh (x+y) \cosh (x-y)
\end{aligned}
$$

one sees that

$$
\tilde{K}(x, y)=\frac{1}{2 \pi}(\operatorname{sech}(x-y)+\operatorname{sech}(x+y)) .
$$

Now $\widetilde{\psi}$ is even, and when $\tilde{K}$ is restricted to the space of even functions it equals the operator with convolution kernel sech $(x-y) / \pi$. Conjugating with the Fourier transform, this operator becomes multiplication by sech $(\pi \xi / 2)$. The Fourier transform of $\operatorname{sech} x$ with factor $1 / 2 \pi$ is $(1 / 2) \operatorname{sech}(\pi \xi / 2)$, and the Fourier transform of $\delta_{0}$ with factor 1 is 1 . Therefore

$$
\left((I+\tilde{K})^{-1} \widetilde{\psi}, \delta_{0}\right)=\frac{1}{2} \int_{-\infty}^{\infty} \frac{1}{1+\operatorname{sech}(\pi \xi / 2)} \operatorname{sech}(\pi \xi / 2) d \xi=\frac{2}{\pi} .
$$

Thus the inner product in (20) is $2 / \pi$ as claimed.

\section{Appendix - The formulas for $\mathfrak{p}(\mathrm{x}, \mathrm{y} ; t)$}

The Weyl group $\mathbb{B}_{n}$ is the group of signed permutations, functions $\sigma:[1, n] \rightarrow$ $[-n,-1] \cup[1, n]$ such that $|\sigma| \in \mathbb{S}_{n}$. An inversion in $\mathbb{B}_{n}$ is a pair $( \pm \sigma(i), \sigma(j))$ with $i<j$ such that $\pm \sigma(i)>\sigma(j)$. We write $\tau=p / q$.

We define

$$
S\left(\xi, \xi^{\prime}\right)=-\frac{p+q \xi \xi^{\prime}-\xi}{p+q \xi \xi^{\prime}-\xi^{\prime}}, \quad r(\xi):=\frac{\xi-1}{1-\tau \xi^{-1}}, \quad \varepsilon(\xi)=p \xi^{-1}+q \xi-1,
$$

and then define

$$
A_{\sigma}=\prod_{\sigma(i)<0} r\left(\xi_{\sigma(i)}\right) \times \prod\left\{S\left(\xi_{a}, \xi_{b}\right):(a, b) \text { is an inversion in } \mathbb{B}_{n}\right\},
$$


with the convention $\xi_{-a}=\tau / \xi_{a}$.

The formula, valid when $q \neq 0$, is

$$
\mathfrak{p}(\mathbf{x}, \mathbf{y} ; t)=\frac{1}{n !} \sum_{\sigma \in \mathbb{B}_{n}} \frac{1}{(2 \pi i)^{n}} \int \cdots \int A_{\sigma}(\xi) \prod_{i}\left(\xi_{\sigma(i)}^{x_{i}} \xi_{i}^{-y_{i}-1} e^{\varepsilon\left(\xi_{i}\right) t}\right) d \xi_{1} \cdots d \xi_{n}
$$

where $\mathbf{x}=\left\{x_{1}, \ldots, x_{n}\right\}$ and $\mathbf{y}=\left\{y_{1}, \ldots, x_{n}\right\}$. The domain of integration is

$$
\bigcup_{\mu \in \mathbb{S}_{n}} \mathcal{C}_{\mu(1)} \times \cdots \times \mathcal{C}_{\mu(n)}
$$

where the $\mathcal{C}_{a}$ are circles with center $1 / 2 q$ and distinct radii $R_{a}$. The $R_{a}$ should be so large that $S\left(\xi, \xi^{\prime}\right)$ is analytic for $\xi, \xi^{\prime}$ on and outside $\mathcal{C}_{a} 14$

In the special case $n=1$,

$$
\mathfrak{p}(x, y ; t)=\frac{1}{2 \pi i} \int_{\mathcal{C}_{R}}\left[\xi^{x-y-1}+\frac{\tau-\xi}{1-\xi} \tau^{x} \xi^{-x-y-2}\right] e^{\varepsilon(\xi) t} d \xi,
$$

where $\mathcal{C}_{R}$ is a circle around 0 of radius $R>1$.

The formulas do not hold for $p=1$ TASEP on $\mathbb{Z}^{+}$. But then the probability is the same as for TASEP on $\mathbb{Z}$, and we have then [3] (or [5, p. 820])

$$
\mathfrak{p}(\mathbf{x}, \mathbf{y} ; t)=\operatorname{det}\left(\int_{\mathcal{C}_{r}}(1-\xi)^{j-i} \xi^{x_{i}-y_{j}-1} e^{t \varepsilon(\xi)} d \xi\right)
$$

where $\mathcal{C}_{r}$ is a circle with center 0 and radius $r<1$.

\section{Acknowledgments}

We thank Lauren Williams for elaborating for us some of the results of [4].

This work was supported by the National Science Foundation through grants DMS-1207995 (first author) and DMS-0854934 (second author).

\section{References}

[1] B. Derrida, M. Evans, V. Hakim, and V. Pasquier, Exact solution of a $1 D$ asymmetric exclusion model using a matrix formulation, J. Phys. A: Math. Gen. 26 (1993), 1493-1517.

\footnotetext{
${ }^{14}$ We cannot simply take $\mathcal{C} \times \cdots \times \mathcal{C}$ with $\mathcal{C}$ a circle with large radius, because then there would be nonintegrable singularities of $S\left(\xi_{a}, \xi_{b}\right)$ on the contour when $a>0, b<0$. However by taking the $R_{a} \rightarrow R$ we can interpret each integral as a symmetric distribution supported on $\mathcal{C} \times \cdots \times \mathcal{C}$ applied to the product in the integrand.
} 
[2] P. L. Krapivsky, Symmetric exclusion process with a localized source, Phys. Rev. E86 (2012), 041103.

[3] G. M. Schütz, Exact solution of the master equation for the asymmetric exclusion process, J. Stat. Phys. 88 (1997), 427-445.

[4] T. Sasamoto and L. Williams, Combinatorics of the asymmetric exclusion process on a semi-infinite lattice, arXiv:1204.1114.

[5] C. A. Tracy and H. Widom, Integral formulas for the asymmetric simple exclusion process, Comm. Math. Phys. 279 (2008), 815-844.

[6] C. A. Tracy and H. Widom, Erratum to "Integral Formulas for the Asymmetric Simple Exclusion Process", Comm. Math. Phys., 304 (2011) 875-878.

[7] C. A. Tracy and H. Widom, The Bose gas and asymmetric simple exclusion process on the half-line, J. Stat. Phys., 150 (2013), 1-12. 